\title{
Observables sensitive to absolute neutrino masses. II
}

\author{
G.L. Fogli ${ }^{1,2}$, E. Lisi ${ }^{2}$, A. Marrone ${ }^{1,2}$, A. Melchiorri ${ }^{3}$, A. Palazzo ${ }^{4}$, A.M. Rotunno ${ }^{1,2}$, P. Serra ${ }^{5}$, J. Silk ${ }^{6}$, A. Slosar ${ }^{7}$ \\ 1 Dipartimento di Fisica, Università di Bari, Via Amendola 173, 70126, Bari, Italy \\ ${ }^{2}$ Istituto Nazionale di Fisica Nucleare (INFN), Sezione di Bari, Via Orabona 4, 70126 Bari, Italy \\ 3 Dipartimento di Fisica and Sezione INFN, Università degli Studi \\ di Roma "La Sapienza", P.le Aldo Moro 5, 00185, Rome, Italy \\ 4 AHEP Group, Institut de Física Corpuscular, CSIC/Universitat de València, \\ Edifici Instituts d'Investigació, Apt. 22085, 46071 València, Spain \\ 5 Department of Physics and Astronomy, University of California, Irvine, CA 92697-4575 \\ ${ }^{6}$ Astrophysics, Denys Wilkinson Building, Keble Road, Oxford, OX1 3RH, United Kingdom \\ 7 Berkeley Center for Cosmological Physics, Physics Department, University of California, Berkeley CA 94720
}

\begin{abstract}
In this followup to Phys. Rev. D 75, 053001 (2007) arXiv:hep-ph/0608060 we report updated constraints on neutrino mass-mixing parameters, in light of recent neutrino oscillation data (KamLAND, SNO, and MINOS) and cosmological observations (WMAP 5-year and other data). We discuss their interplay with the final $0 \nu 2 \beta$ decay results in ${ }^{76} \mathrm{Ge}$ claimed by part of the HeidelbergMoscow Collaboration, using recent evaluations of the corresponding nuclear matrix elements, and their uncertainties. We also comment on the $0 \nu 2 \beta$ limits in ${ }^{130}$ Te recently set by Cuoricino, and on prospective limits or signals from the KATRIN experiment.

PACS numbers: 14.60.Pq, 23.40.-s, 95.35.+d, 98.80.-k
\end{abstract}

Introduction. This paper is meant as a followup to the article [1] where, building upon previous work [2], we presented constraints on the neutrino mass-squared differences $\left(\delta m^{2}, \Delta m^{2}\right)$ and mixing angles $\left(\sin ^{2} \theta_{12}, \sin ^{2} \theta_{23}, \sin ^{2} \theta_{13}\right)$, as well as on three observables sensitive to absolute $\nu$ masses: the effective mass $m_{\beta}$ in single beta decay, the effective Majorana mass $m_{\beta \beta}$ in neutrinoless double beta $(0 \nu 2 \beta)$ decay, and the sum of $\nu$ masses $\Sigma$ in cosmology - see [1, 2, 3] for notation and conventions. We update the results of [1] by including several new experimental inputs, largely presented or discussed at the recent Neutrino 2008 Conference [4].

Neutrino oscillation updates. The Kamioka Liquid Scintillator Anti-Neutrino Detector (KamLAND) Collaboration has presented reactor $\bar{\nu}_{e}$ disappearance and geo- $\nu$ results for an exposure of $2.881 \mathrm{kTy}$ [5], a factor $\sim 4$ higher than the one we used in [1]. Following [5], the KamLAND spectrum analysis in [1, 3] has been upgraded [6] to include the rates of geo- $\nu$ events from $\mathrm{U}$ and Th decay as low-energy nuisance parameters.

Results from the third phase of the Sudbury Neutrino Observatory (SNO-III) 7], recently presented at Neutrino 2008 [4], have been included [6] in the form of two new integral determinations of the charged- and neutral-current event rates [7]. Other solar $\nu$ updates, with a minor impact in the global parameter estimate, include the latest Borexino results [8] and reevaluated GALLEX data [9] — see also [10].

The Main Injector Neutrino Oscillation Search (MINOS) Collaboration has presented accelerator $\nu_{\mu}$ disappearance data from $3.36 \times 10^{20}$ protons on target [1], a factor of $\sim 2.6$ larger than previously used in [1]. In the official MINOS data analysis [11], for any given energy profile of the $\nu_{\mu}$ survival probability $P_{\mu \mu}\left(E_{\nu}\right)$, a "beam matrix" method is used to map the energy spectrum from near to far, and an independent near-far extrapolation method is used as a cross-check [12]. This approach can be fully implemented only within the Collaboration. For our purposes, we analyze the 18-bin energy spectrum ratio [11] by folding the function $P_{\mu \mu}\left(E_{\nu}, \Delta m^{2}, \sin ^{2} \theta_{23}, \sin ^{2} \theta_{13}\right)$, with empirical energy resolution profiles, which mimic the near-far energy spectrum mapping of [12]. Normalization and energy scale systematics are treated as nuisance parameters.

In the limit $\theta_{13} \rightarrow 0$, our effective $2 \nu$ parameter fits reproduce very well the official ones as obtained by the KamLAND [5], SNO-III [7], and MINOS [1] Collaborations. In our global analysis, however, we treat $\theta_{13}$ as a free parameter.

Figure 1 displays our updated results on the mass-mixing parameters, in terms of standard deviations $n_{\sigma}$ from the best fit $\left(n_{\sigma}=\sqrt{\Delta \chi^{2}}\right.$ after $\chi^{2}$ marginalization). Table \ summarizes such results in numerical form. As compared with [1], the $\Delta m^{2}$ uncertainty is almost halved (by new MINOS data), and both the $\delta m^{2}$ and the $\sin ^{2} 2 \theta_{12}$ allowed ranges are reduced (by new KamLAND and SNO data). The range of $\sin ^{2} \theta_{23}$ is almost unchanged. As discussed in [10], an intriguing new result is the preference for $\theta_{13}>0$ at the level of $\sim 1.6 \sigma$ (or, equivalently, $\sim 90 \%$ C.L.). Such an indication emerges from the combination of two independent hints in favor of $\theta_{13}>0$, each at the level of $\sim 1 \sigma$ : an 
TABLE I: Global $3 \nu$ oscillation analysis (2008): best-fit values and allowed $n_{\sigma}$ ranges for the mass-mixing parameters.

\begin{tabular}{cccccc}
\hline \hline Parameter & $\delta m^{2} / 10^{-5} \mathrm{eV}^{2}$ & $\sin ^{2} \theta_{12}$ & $\sin ^{2} \theta_{13}$ & $\sin ^{2} \theta_{23}$ & $\Delta m^{2} / 10^{-3} \mathrm{eV}^{2}$ \\
\hline Best fit & 7.67 & 0.312 & 0.016 & 0.466 & 2.39 \\
$1 \sigma$ range & $7.48-7.83$ & $0.294-0.331$ & $0.006-0.026$ & $0.408-0.539$ & $2.31-2.50$ \\
$2 \sigma$ range & $7.31-8.01$ & $0.278-0.352$ & $<0.036$ & $0.366-0.602$ & $2.19-2.66$ \\
$3 \sigma$ range & $7.14-8.19$ & $0.263-0.375$ & $<0.046$ & $0.331-0.644$ & $2.06-2.81$ \\
\hline \hline
\end{tabular}

older one, found in the atmospheric $\nu$ data analysis of [3], and a newer one, coming from the small difference between the best-fit values of $\sin ^{2} 2 \theta_{12}$ in KamLAND [5] and SNO [7] - a difference which is reduced for $\sin ^{2} \theta_{13} \sim$ few $\%$. Hereafter, as in [1, 2], we shall show results at a conservative $2 \sigma(95 \%)$ confidence level, in which case only an upper bound can be placed on $\theta_{13}$.

Figure 2 shows the $2 \sigma$ bounds implied by the above $\nu$ oscillation parameter constraints (for normal or inverted hierarchy) in the three planes charted by any two among the three observables $\left(m_{\beta}, m_{\beta \beta}, \Sigma\right)$. A measurement of any such quantity, coupled with the bounds in Fig. 2, provides "predictions" for the other two quantities [1, 2, 3].

Cosmology updates. Within the standard cosmological model, the 5-year data recently released by Wilkinson Microwave Anisotropy Probe (WMAP 5y) [13, 14] constrain, by themselves, the sum of the $\nu$ masses $\Sigma$ below 1.3 eV at $95 \%$ C.L. 13]. This limit can be strengthened in the sub-eV range by adding further cosmological data; for instance, the WMAP collaboration finds $\Sigma<0.61 \mathrm{eV}$ by adding Baryonic Acoustic Oscillation (BAO) and type-Ia supernova (SN-Ia) data [14].

We consider five representative combinations of cosmological data, which lead to increasingly stronger upper limits on $\Sigma$ : (1) Cosmic Microwave Background (CMB) anisotropy data from: WMAP 5y [14], Arcminute Cosmology Bolometer Array Receiver (ACBAR) [15], Very Small Array (VSA) 16], Cosmic Background Imager (CBI) 17] and BOOMERANG [18] experiments; (2) the above CMB results plus the large-scale structure (LSS) information on galaxy clustering coming from the Luminous Red Galaxies Sloan Digital Sky Survey (SDSS) [19]; (3) the above CMB results plus the Hubble Space Telescope (HST) prior on the value of the reduced Hubble constant, $h=0.72 \pm 0.07$ [20], and the luminosity distance SN-Ia data of [21]; (4) the data in (3) plus the BAO data from [22]; (5) the data in (4), plus the small scale primordial spectrum from Lyman-alpha (Ly $\alpha$ ) forest clouds [23, 24].

Adopting the same procedure described in [1], based on the publicly available COSMOMC code 25], we find the upper limits on $\Sigma$ summarized in Table II and in Fig. 3. The bound in Table If for case (1) is dominated by WMAP-5y data. The results for cases (1) and (4) are in agreement with similar constraints presented in [13] and [14], respectively, even if the datasets considered here for BAO and SN-Ia are different. In Fig. 3, the slight preference for $\Sigma \neq 0$ at best fit for case (4) [and case (1)], also found in [14], is not statistically significant. As in Ref. [1], we find that Ly $\alpha$ data have a strong impact on $\Sigma$, but their inclusion in global fits is debated [14] due to systematics still under scrutiny. The upper limits from cases (1)-(4) (namely, $\Sigma<0.6-1.2 \mathrm{eV}$ ) should be considered as more conservative. Including LSS data would not significantly modify case 5 , which is dominated by Ly $\alpha$ data. In the following, we shall focus on the two extreme cases (1) and (5).

$0 \nu 2 \beta$ decay updates. The final analysis of part of the Heidelberg-Moscow (HM) Collaboration reports a $0 \nu 2 \beta$ signal in ${ }^{76} \mathrm{Ge}$ with half-life $T_{1 / 2}^{0 \nu}=2.23_{-0.31}^{+0.44} \times 10^{25}$ y $(1 \sigma$ errors $)$ at a claimed C.L. $>6 \sigma$ [26]. The previously estimated $T_{1 / 2}^{0 \nu}$ [27], as used in [1], was a factor of $\sim 2$ smaller. The claim is controversial, but the experimental sensitivity to the signal (if real) is no longer questioned [28].

From a theoretical viewpoint, the $0 \nu 2 \beta$ nuclear matrix elements (NME) $C_{m m}$ and uncertainties estimated via Quasiparticle Random Phase Approximations (QRPA) in 29] (as used in [1]) have been recently revised [30, 31],

TABLE II: Representative cosmological data sets and corresponding $2 \sigma$ (95\% C.L.) constraints on the sum of $\nu$ masses $\Sigma$.

\begin{tabular}{llr}
\hline \hline Case & Cosmological data set & $\Sigma($ at $2 \sigma)$ \\
\hline 1 & CMB & $<1.19 \mathrm{eV}$ \\
2 & $\mathrm{CMB}+\mathrm{LSS}$ & $<0.71 \mathrm{eV}$ \\
3 & $\mathrm{CMB}+\mathrm{HST}+\mathrm{SN}-\mathrm{Ia}$ & $<0.75 \mathrm{eV}$ \\
4 & $\mathrm{CMB}+\mathrm{HST}+\mathrm{SN}-\mathrm{Ia}+\mathrm{BAO}$ & $<0.60 \mathrm{eV}$ \\
5 & $\mathrm{CMB}+\mathrm{HST}+\mathrm{SN}-\mathrm{Ia}+\mathrm{BAO}+\mathrm{Ly} \alpha$ & $<0.19 \mathrm{eV}$ \\
\hline \hline
\end{tabular}


especially to improve the so-called short-range correlations. We adopt for $C_{m m}$ the central values and errors of [31], which agree with independent QRPA [32, 33] and shell model [34] evaluations within $\sim 2 \sigma$ (see Fig. 11 of [31] and related comments therein).

The effect of both the $T_{1 / 2}^{0 \nu}$ and the NME updates for ${ }^{76} \mathrm{Ge}$ is to lower the central value - and to enlarge the errorsof the effective mass parameter $m_{\beta \beta}^{2}=m_{e}^{2} / C_{m m} T_{1 / 2}^{0 \nu}$. By taking logs (in base 10) to linearize error propagation, we have $\log \left(T_{1 / 2}^{0 \nu} / \mathrm{y}\right)=23.35 \pm 0.16(2 \sigma)$ from [26] and $\log \left(C_{m m} / \mathrm{y}^{-1}\right)=-12.82 \pm 0.48(2 \sigma)$ from [31], so that

$$
\log \left(m_{\beta \beta} / \mathrm{eV}\right)=-0.54 \pm 0.26 \quad(\mathrm{HM} \text { claim, } 2 \sigma),
$$

where the experimental error and the (dominant) theoretical error have been added in quadrature.

The Cuoricino experiment, which does not find $0 \nu 2 \beta$ decay signals in ${ }^{130} \mathrm{Te}$, quotes $T_{1 / 2}^{0 \nu}>3.1 \times 10^{24} \mathrm{y}$ at $90 \%$ C.L. [28], or $T_{1 / 2}^{0 \nu}>2.5 \times 10^{24}$ y at $95 \%$ C.L. [35]. Using the latter limit as $\log \left(T_{1 / 2}^{0 \nu} / \mathrm{y}\right)>24.4$, and the ${ }^{130} \mathrm{Te}$ NME estimate $\log \left(C_{m m} / \mathrm{y}^{-1}\right)=-12.27 \pm 0.28(2 \sigma)$ from [31], we get

$$
\log \left(m_{\beta \beta} / \mathrm{eV}\right)<[-0.63,-0.07] \quad(\text { Cuoricino, } 2 \sigma)
$$

where the range due to the $2 \sigma$ uncertainty of the NME is explicitly reported.

A comparison of the corresponding $m_{\beta \beta}$ ranges $(2 \sigma)$,

$$
\begin{aligned}
0.16 & <m_{\beta \beta} / \mathrm{eV}<0.52 & & \text { (HM claim) } \\
0 & \leq m_{\beta \beta} / \mathrm{eV}<0.23 & & \text { (Cuoricino, "favorable" NME) } \\
0 & \leq m_{\beta \beta} / \mathrm{eV}<0.85 & & \text { (Cuoricino, "unfavorable" NME) }
\end{aligned}
$$

shows that current Cuoricino data may or may not disfavor a fraction of the HM range for $m_{\beta \beta}$ at $2 \sigma$, depending on the (still quite uncertain) value of the ${ }^{130} \mathrm{Te} 0 \nu 2 \beta$ NME. A similar conclusion (albeit with somewhat different preferred ranges for $m_{\beta \beta}$ ) has been reached in [28]. Therefore, the $0 \nu 2 \beta$ claim [26] remains an open issue at present, and we shall consider the possibility that it corresponds to a real signal.

Discussion. Figure 4 shows the regions allowed at $2 \sigma$ in normal and inverted hierarchy (slanted bands) by the combination of oscillation results with the first dataset in Table II (CMB), in the plane spanned by $\left(\Sigma, m_{\beta \beta}\right)$. This is the most conservative case, with the weakest limits on $\Sigma$, and the largest overlap between the regions separately allowed by oscillation $+\mathrm{CMB}$ data and by the $0 \nu 2 \beta$ claim. The results of a global $\chi^{2}$ fit are shown as a thick black wedge in the upper right part of the figure. [The combination includes the current limit $m_{\beta}<1.8 \mathrm{eV}(2 \sigma)$ [1] which, however, provides only a minor contribution.] Such global combination would correspond to nearly degenerate masses in the range

$$
m_{1} \simeq m_{2} \simeq m_{3} \in[0.15,0.46] \mathrm{eV} \quad(2 \sigma) .
$$

In this case (degenerate spectrum), the preferred range for effective neutrino mass in $\beta$ decay would also be $m_{\beta} \in[0.15,0.46] \mathrm{eV}$. In the upper half of this range, the KArlsruhe TRItium Neutrino (KATRIN) $\beta^{-}$experiment could make a $5 \sigma$ discovery, according to the estimated sensitivity [36]. A $3 \sigma$ evidence could still be found in KATRIN for $m_{\beta} \sim 0.3 \mathrm{eV}$. Below this value, the sensitivity would be rapidly degraded, and only upper bounds could be placed for $m_{\beta} \lesssim 0.2 \mathrm{eV}[36]$. The possibility of reaching a $\sim 0.1-0.2 \mathrm{eV}$ sensitivity with a different approach to $\beta$ decay is being discussed [37].

If the cosmological dataset (1) were replaced by the datasets (2)-(4) in Table II the overlap region between the $0 \nu 2 \beta$ band and the oscillation+cosmological bands in Fig. 4 would shrink (not shown), but would not disappear. Therefore, within the standard $3 \nu$ framework and the present uncertainties, the $0 \nu 2 \beta$ claim clashes with oscillation + cosmological data only if the latter include $\operatorname{Ly} \alpha$ data.

Figure 5 is analogous to Fig. 4, but refers to the fifth dataset in Table I (all cosmological data, including Ly $\alpha$ ). In this case, the allowed regions do not overlap and cannot be combined, since the relatively strong cosmological limit $\Sigma<0.19 \mathrm{eV}$ implies $m_{\beta \beta} \lesssim 0.08 \mathrm{eV}$, in contradiction with Eq. (3). Solutions to this discrepancy would require that either some data or their interpretation are wrong.

In conclusion, important pieces of information are being slowly added to the puzzle of absolute $\nu$ masses. In this followup to [1], we have discussed the most recent oscillation and nonoscillation updates in the field, after the recent Neutrino 2008 Conference [4]. Oscillation parameters are robustly constrained, and an intriguing indication for $\theta_{13}>0$ emerges, as summarized in Fig. 1 and in Table [. Concerning nonoscillation observables, despite some recent experimental and theoretical progress, a coherent picture remains elusive. In particular, the $0 \nu 2 \beta$ claim is still under independent experimental scrutiny, and it may be compatible (Fig. 4) or incompatible (Fig. 5) with the cosmological bounds (Table III), depending on data selection (especially Ly $\alpha$ ). A confident assessment of the $\nu$ mass scale will require converging evidence from at least two of the three observables $\left(m_{\beta}, m_{\beta \beta}, \Sigma\right)$ within the bands allowed by oscillation data in Fig. 2 . 
Acknowledgments. G.L. Fogli, E. Lisi, A. Marrone, and A. Rotunno acknowledge support by the Italian MIUR and INFN through the "Astroparticle Physics" research project, and by the EU ILIAS through the ENTApP project. A. Palazzo is supported by MEC under the I3P program, by Spanish grants FPA2005-01269 and by European Commission network MRTN-CT-2004-503369 and ILIAS/N6 RII3-CT-2004-506222. This research has also been supported by ASI contract I/016/07/0 "COFIS."

[1] G. L. Fogli, E. Lisi, A. Marrone, A. Melchiorri, A. Palazzo, P. Serra, J. Silk, and A. Slosar, "Observables sensitive to absolute neutrino masses: A reappraisal after WMAP-3y and first MINOS results," Phys. Rev. D 75, 053001 (2007) arXiv:hep-ph/0608060.

[2] G. L. Fogli, E. Lisi, A. Marrone, A. Melchiorri, A. Palazzo, P. Serra and J. Silk, "Observables sensitive to absolute neutrino masses: Constraints and correlations from world neutrino data," Phys. Rev. D 70, 113003 (2004) arXiv:hep-ph/0408045.

[3] G. L. Fogli, E. Lisi, A. Marrone and A. Palazzo, "Global analysis of three-flavor neutrino masses and mixings," Prog. Part. Nucl. Phys. 57, 742 (2006) arXiv:hep-ph/0506083.

[4] Neutrino 2008, XXIII International Conference on Neutrino Physics and Astrophysics (Christchurch, New Zealand, 2008). Webpage: www2.phys.canterbury.ac.nz/ jaa53

[5] KamLAND Collaboration, S. Abe et al., "Precision Measurement of Neutrino Oscillation Parameters with KamLAND," Phys. Rev. Lett. 100, 221803 (2008) arXiv:0801.4589v3 [hep-ex]].

[6] G. L. Fogli, E. Lisi, A. Palazzo, and A. M. Rotunno, preprint in preparation.

[7] SNO Collaboration, "An Independent Measurement of the Total Active ${ }^{8}$ B Solar Neutrino Flux Using an Array of ${ }^{3}$ He Proportional Counters at the Sudbury Neutrino Observatory," arXiv:0806.0989 [nucl-ex].

[8] Borexino Collaboration, C. Arpesella et al., "New results on solar neutrino fluxes from 192 days of Borexino data," arXiv:0805.3843 [astro-ph].

[9] F. Kaether, "Data Analysis of the solar neutrino experiment GALLEX" (PhD Thesis, Heidelberg, 2007; advisor: W. Hampel).

[10] G. L. Fogli, E. Lisi, A. Marrone, A. Palazzo and A. M. Rotunno, "Hints of $\theta_{13}>0$ from global neutrino data analysis," arXiv:0806.2649 [hep-ph].

[11] MINOS Collaboration, P. Adamson et al., "Measurement of Neutrino Oscillations with the MINOS Detectors in the NuMI Beam," arXiv:0806.2237 [hep-ex].

[12] MINOS Collaboration, P. Adamson et al., "A Study of Muon Neutrino Disappearance Using the Fermilab Main Injector Neutrino Beam," arXiv:0711.0769 [hep-ex].

[13] WMAP Collaboration, J. Dunkley et al., "Five-Year Wilkinson Microwave Anisotropy Probe Observations: Likelihoods and Parameters from the WMAP data," arXiv:0803.0586 [astro-ph].

[14] WMAP Collaboration, E. Komatsu et al., "Five-Year Wilkinson Microwave Anisotropy Probe Observations: Cosmological Interpretation," arXiv:0803.0547 [astro-ph].

[15] ACBAR Collaboration, C. L. Reichardt et al., "High resolution CMB power spectrum from the complete ACBAR data set," arXiv:0801.1491 [astro-ph].

[16] VSA Collaboration, C. Dickinson et al., "High sensitivity measurements of the CMB power spectrum with the extended Very Small Array," Mon. Not. Roy. Astron. Soc. 353, 732 (2004) arXiv:astro-ph/0402498.

[17] CBI Collaboration, A. C. S. Readhead et al., "Extended Mosaic Observations with the Cosmic Background Imager," Astrophys. J. 609, 498 (2004) arXiv:astro-ph/0402359.

[18] BOOMERANG Collaboration, C. J. MacTavish et al., "Cosmological parameters from the 2003 flight of BOOMERANG," Astrophys. J. 647, 799 (2006) arXiv:astro-ph/0507503.

[19] SDSS Collaboration, M. Tegmark et al., "Cosmological Constraints from the SDSS Luminous Red Galaxies," Phys. Rev. D 74 (2006) 123507 arXiv:astro-ph/0608632.

[20] HST Collaboration, W. L. Freedman et al. "Final Results from the Hubble Space Telescope Key Project to Measure the Hubble Constant," Astrophys. J. 553, 47 (2001) arXiv:astro-ph/0012376.

[21] SNLS Collaboration, P. Astier et al. "The Supernova Legacy Survey: Measurement of $\Omega_{M}, \Omega_{\Lambda}$ and $w$ from the First Year Data Set," Astron. Astrophys. 447, 31 (2006) arXiv:astro-ph/0510447.

[22] SDSS Collaboration, D. J. Eisenstein et al., "Detection of the Baryon Acoustic Peak in the Large-Scale Correlation Function of SDSS Luminous Red Galaxies," Astrophys. J. 633, 560 (2005) arXiv:astro-ph/0501171.

[23] P. McDonald et al., Astrophys. J. Suppl. 163, 80 (2006).

[24] P. McDonald et al., Astrophys. J. 635, 761 (2005).

[25] A. Lewis and S. Bridle, "Cosmological parameters from CMB and other data: a Monte-Carlo approach," Phys. Rev. D 66, 103511 (2002) arXiv:astro-ph/0205436. Available at cosmologist.info.

[26] H. V. Klapdor-Kleingrothaus and I. V. Krivosheina, "The Evidence For The Observation Of $0 \nu \beta \beta$ Decay: The Identification Of $0 \nu \beta \beta$ Events From The Full Spectra," Mod. Phys. Lett. A 21, 1547 (2006).

[27] H. V. Klapdor-Kleingrothaus, I. V. Krivosheina, A. Dietz and O. Chkvorets, "Search for neutrinoless double beta decay with enriched Ge-76 in Gran Sasso 1990-2003," Phys. Lett. B 586, 198 (2004) arXiv:hep-ph/0404088.

[28] C. Arnaboldi et al., "Results from the CUORICINO neutrinoless double beta decay experiment," arXiv:0802.3439 [hep-ex].

[29] V. A. Rodin, A. Faessler, F. Simkovic and P. Vogel, "Assessment of uncertainties in QRPA 0 $\nu \beta \beta$-decay nuclear matrix 
elements," Nucl. Phys. A 766, 107 (2006) arXiv:nucl-th/0503063v3].

[30] V. A. Rodin, A. Faessler, F. Simkovic and P. Vogel, Erratum to [29], Nucl. Phys. A 793, 213 (2007) arXiv:0706.4304] [nucl-th]].

[31] F. Simkovic, A. Faessler, V. Rodin, P. Vogel, and J. Engel, "Anatomy of nuclear matrix elements for neutrinoless doublebeta decay," Phys. Rev. C 77, 045503 (2008) arXiv:0710.2055 [nucl-th]].

[32] M. Kortelainen and J. Suhonen, "Improved short-range correlations and $0 \nu \beta \beta$ nuclear matrix elements of ${ }^{76}$ Ge and ${ }^{82}$ Se," Phys. Rev. C 75, 051303 (2007) arXiv:0705.0469 [nucl-th]].

[33] M. Kortelainen and J. Suhonen, "Nuclear matrix elements of neutrinoless double beta decay with improved short-range correlations," Phys. Rev. C 76, 024315 (2007) arXiv:0708.0115 [nucl-th]].

[34] E. Caurier, J. Menendez, F. Nowacki, and A. Poves, "The influence of pairing on the nuclear matrix elements of the neutrinoless double beta decays," Phys. Rev. Lett. 100, 052503 (2008) arXiv:0709.2137 [nucl-th]].

[35] O. Cremonesi, private communication.

[36] C. Weinheimer, "Neutrino mass from beta decay," Proceedings of NOW 2006, Neutrino Oscillation Workshop (Conca Specchiulla, Lecce, Italy, 2006), ed. by P. Bernardini, G.L. Fogli, and E. Lisi, Nucl. Phys. Proc. Suppl. 168, 5 (2007).

[37] A. Nucciotti, "The MARE Project," Proceedings of LT-12, XII International Workshop on Low Temperature Dectectors (Paris, France, 2007), ed. by M. Chapellier and G. Chardin, J. Low Temp. Phys. 151, 597 (2008). 


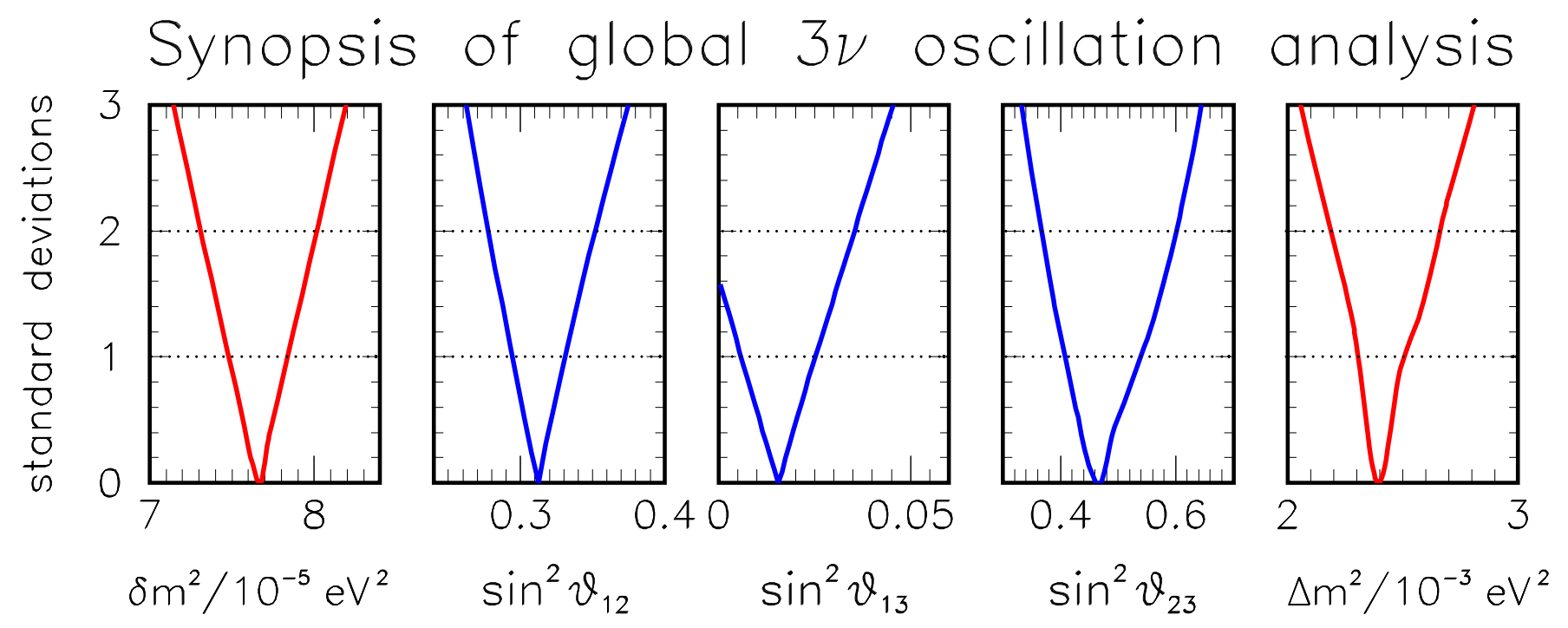

FIG. 1: Global 3 $\nu$ oscillation analysis (2008): Bounds on the mass-mixing oscillation parameters, in terms of standard deviations from the best fit. Note the $1.6 \sigma$ preference for $\theta_{13}>0$. 

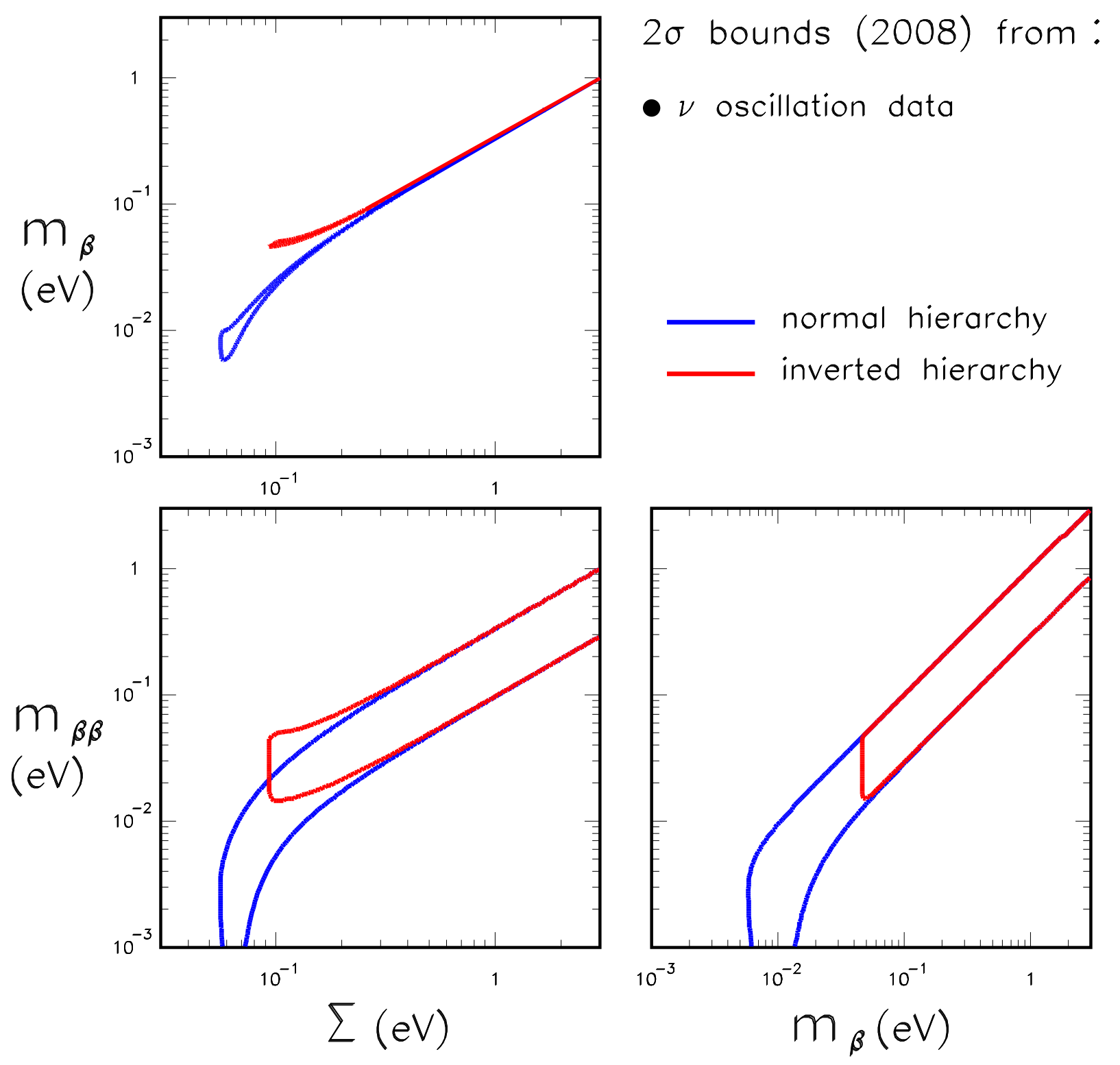

FIG. 2: Bands allowed at $2 \sigma$ by neutrino oscillation data, in each of the three coordinate planes of the parameter space $\left(m_{\beta}, m_{\beta \beta}, \Sigma\right)$, for both normal and inverted hierarchy. 


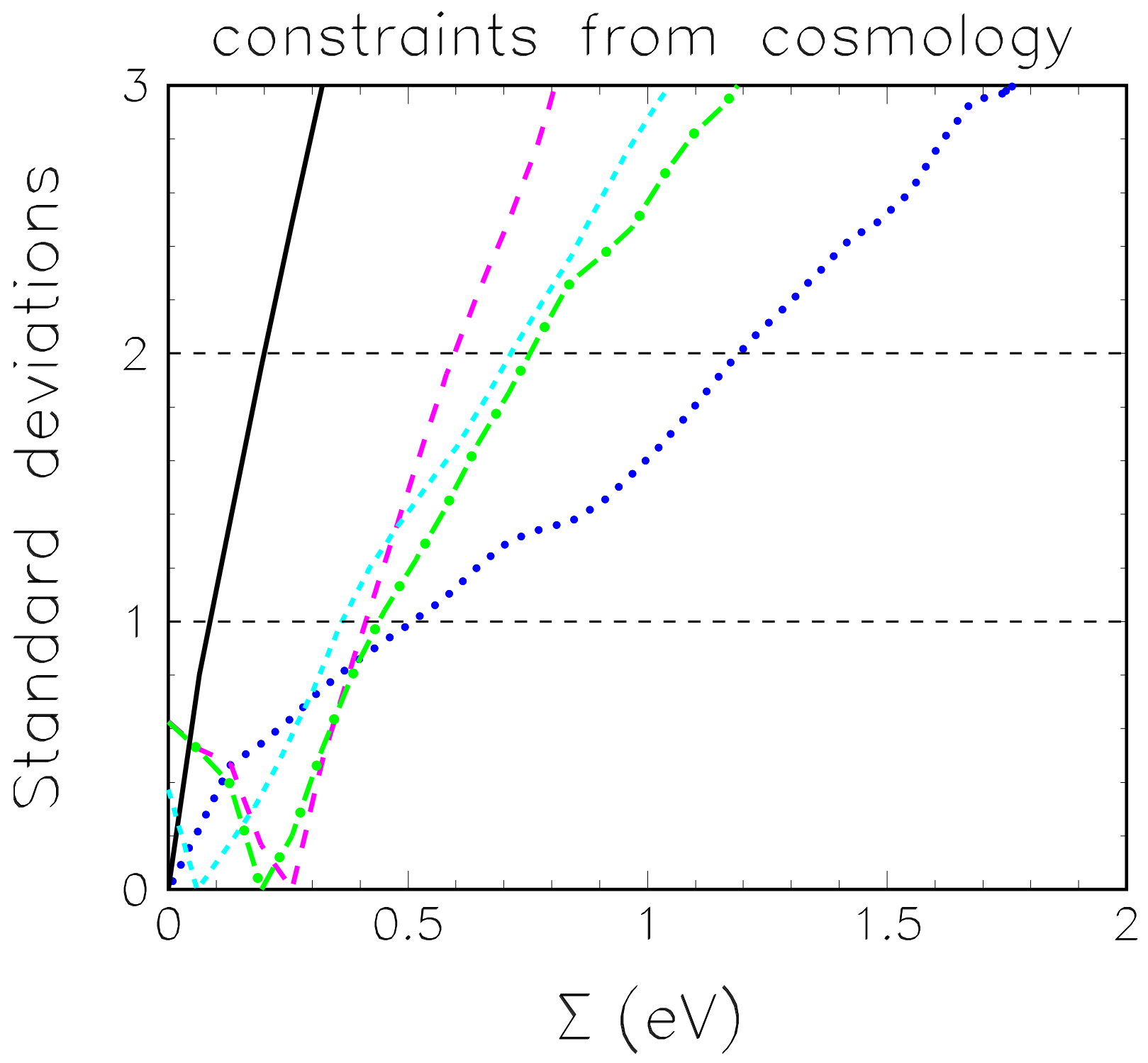

FIG. 3: Cosmological constraints on the sum of neutrino masses $(\Sigma)$. Standard deviation curves for the five datasets in Table II 1 (dotted), 2 (dashed), 3 (dot-dashed), 4 (long dashed), and 5 (solid). 


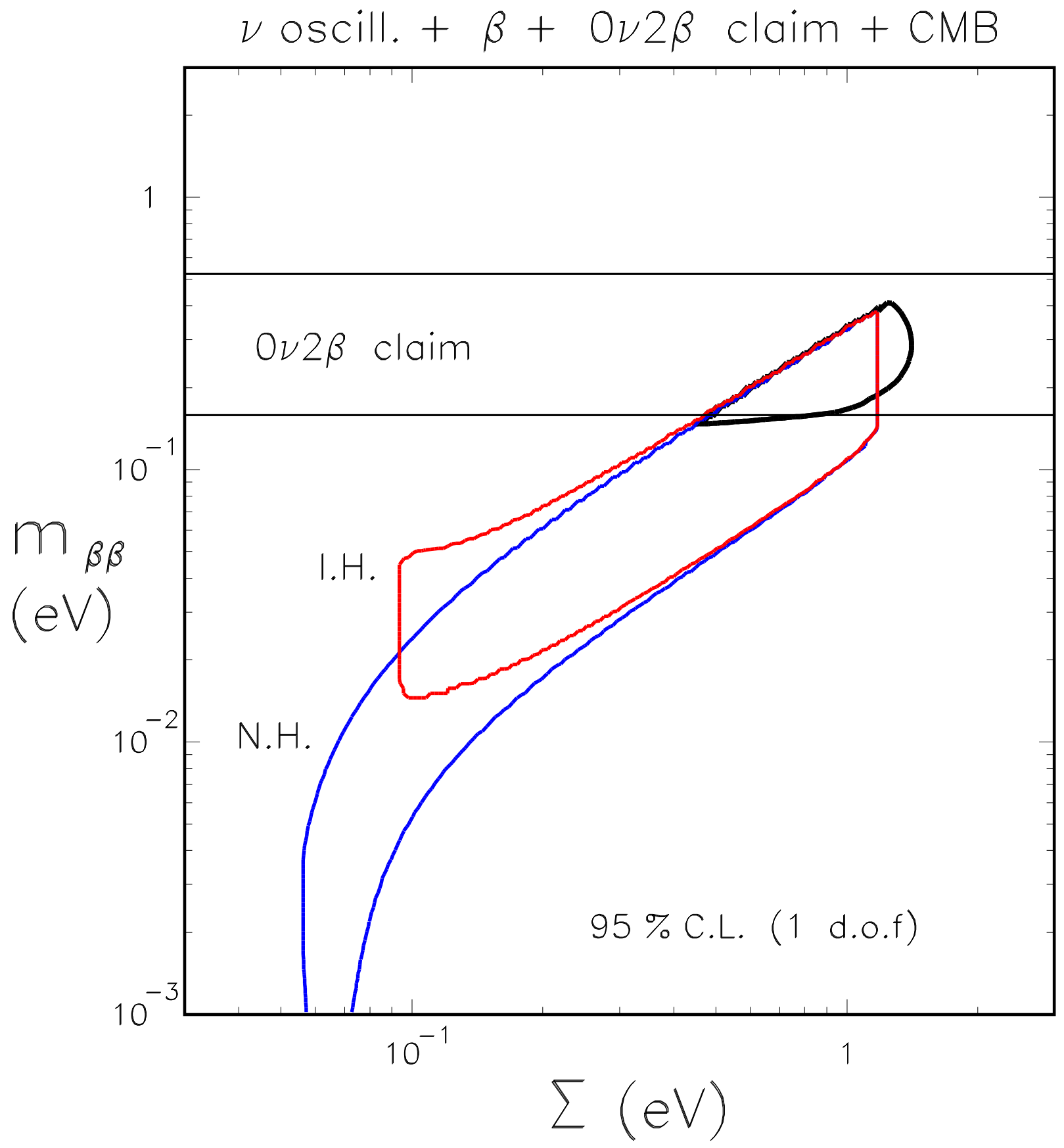

FIG. 4: Global combination of oscillation plus CMB data (case 1 in Table $\amalg$ ) with the $0 \nu 2 \beta$ decay claim, in the plane $\left(\Sigma, m_{\beta \beta}\right)$. 


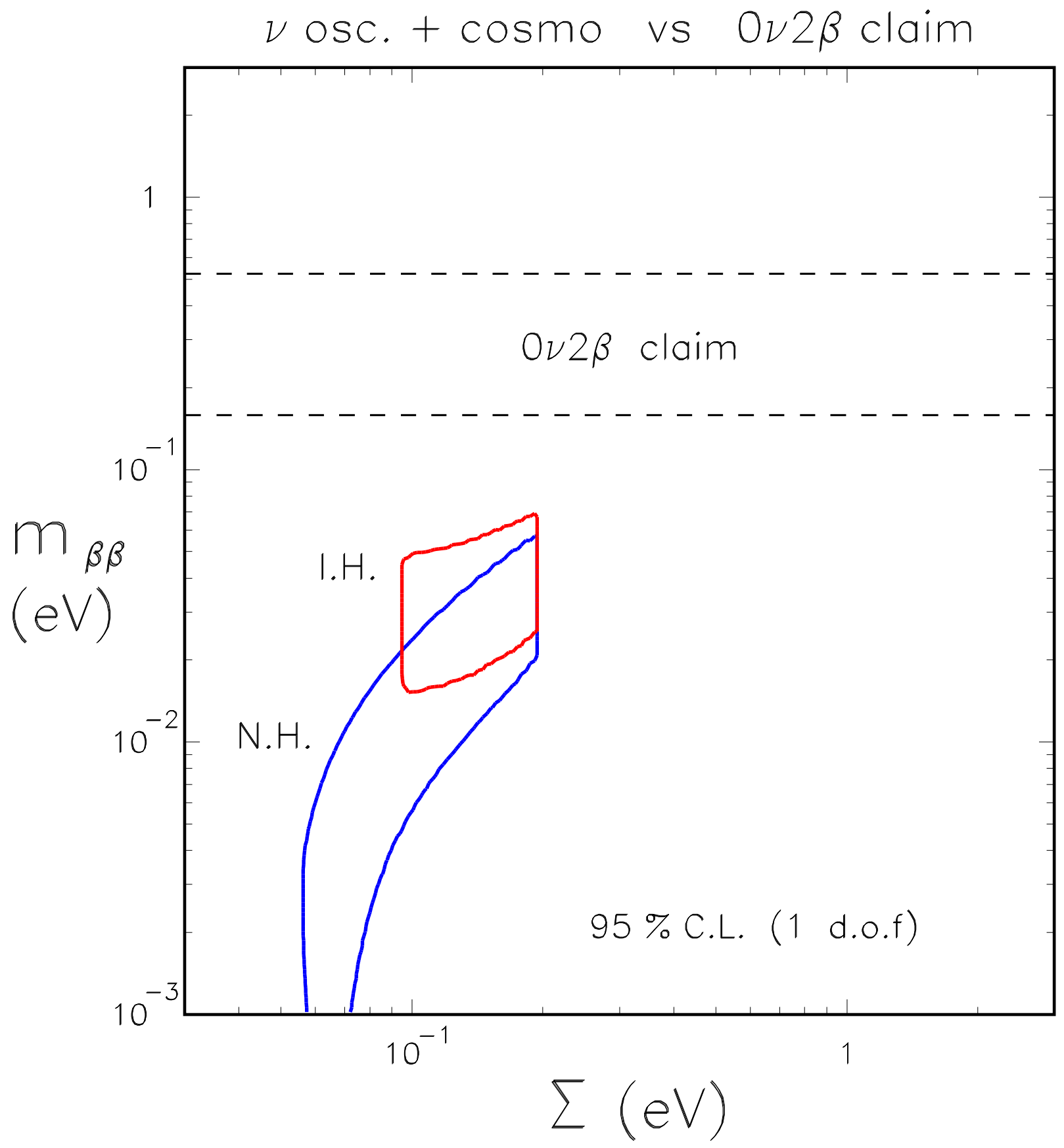

FIG. 5: Bounds from oscillation plus all cosmological data (case 5 in Table II), contrasted with the $0 \nu 2 \beta$ decay claim, in the plane $\left(\Sigma, m_{\beta \beta}\right)$. 